Document downloaded from:

http://hdl.handle.net/10251/143620

This paper must be cited as:

Giner-Sanz, JJ.; Ortega Navarro, EM.; García Gabaldón, M.; Pérez-Herranz, V. (12-0). Algorithm for Assessing the Convergence of a Cyclic Voltammetry to Its Limit Cycle. Journal of The Electrochemical Society. 166(6):H224-H232. https://doi.org/10.1149/2.1111906jes

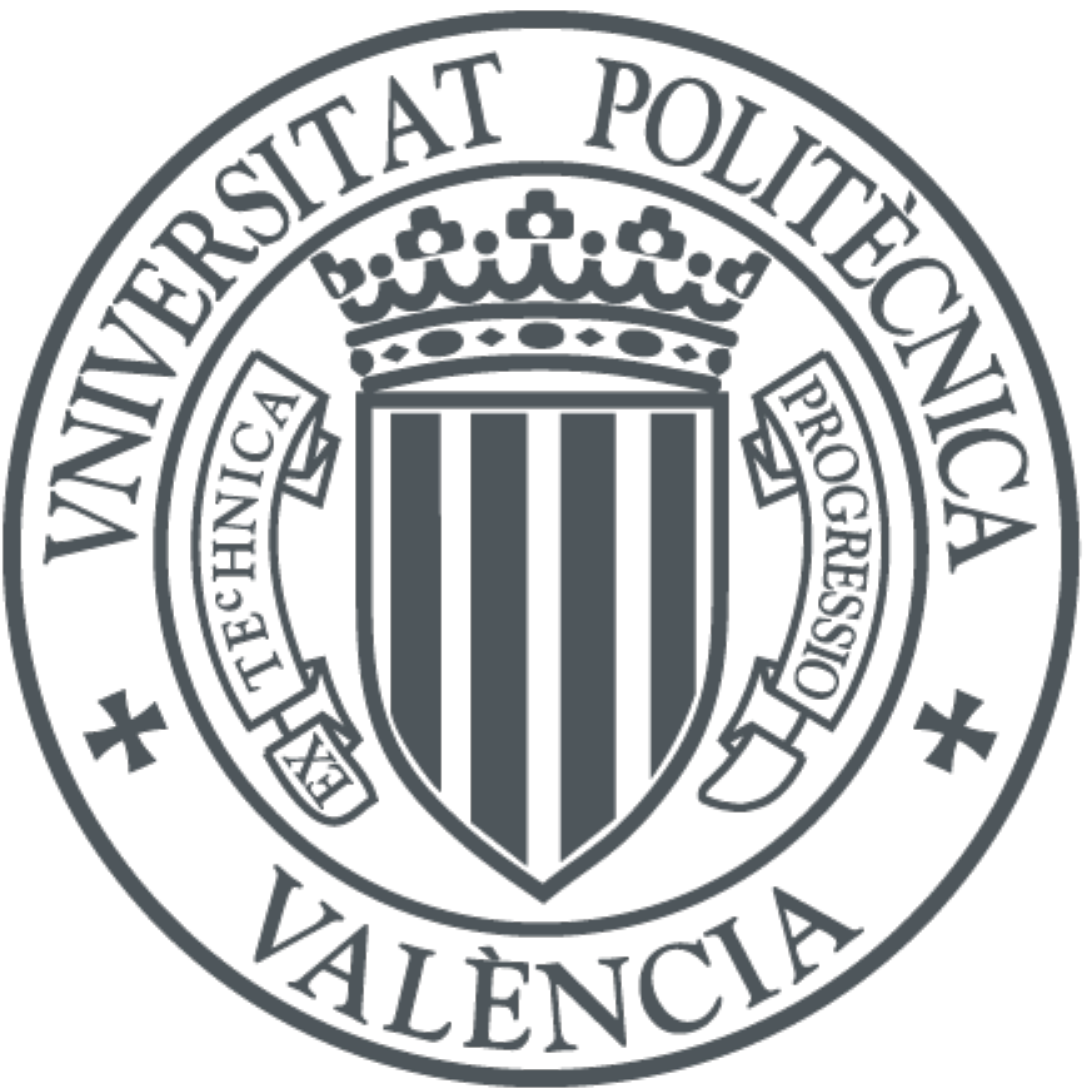

The final publication is available at

https://doi.org/10.1149/2.1111906jes

Copyright The Electrochemical Society

Additional Information 


\title{
Algorithm for assessing the convergence of a cyclic voltammetry to its limit cycle
}

\author{
J. J. Giner-Sanz, E. M. Ortega, M. García-Gabaldón, V. Pérez-Herranz* \\ IEC group, Depto. Ingeniería Química y Nuclear, Universitat Politècnica de València \\ Camino de Vera S/N, 46022 Valencia, Spain \\ *Corresponding author. Tel.: +34-96-3877632; fax: +34-96-3877639; \\ E-mail address: vperez@iqn.upv.es (V.Pérez-Herranz)
}

\begin{abstract}
:
Cyclic voltammetry is one of today's standard electrochemical measurement techniques. What characterises cyclic voltammetry is that potential is linearly ramped in cycles. In general, in this kind of measurements, the system tends to a stationary state, which is known as limit cycle. The common practice for assessing the voltammogram convergence is to perform a multicycle cyclic voltammetry, and visually compare the sequential cycles in order to see if there are significant changes from one cycle to the following one. The main limitation of visual comparison is its limited accuracy and its dependence on the analyst's subjectivity. In this work, an algorithm for quantitatively assessing the convergence of experimental cyclic voltammograms (CVs) was developed. The algorithm was successfully validated experimentally using two systems: it is able to determine whether the CV converged to its limit cycle, and when it converged. Moreover, the algorithm is able to quantify the measurement noise. The low computational cost of the developed algorithm allows to execute it in real time during the cyclic voltammetry measurement. In this way, it can be used in order to automate the measurement process which would decide, according to predefined convergence criteria, when to stop cycling.
\end{abstract}

Keywords: Algorithm, Automated measurements, Convergence assessment, Cyclic voltammetry, Limit Cycle. 


\section{Introduction}

From an historical point of view, the branch of electrochemistry known today as voltammetry, was born with the polarography discovery in 1922, by the Czech chemist Jaroslav Heyrovsky [1]. Discovery for which he received the Nobel Prize in chemistry in 1959. Early voltammetric methods presented a substantial number of limitations that prevented their widespread use. However, in the decades of the 60s and the 70s, significant advances were made in the theory and the instrumentation of voltammetric methods, such as the development of low-cost operational amplifiers that enabled affordable potentiostats [2]. These advances boosted the practical applicability of voltammetric methods, leading to the actual situation where voltammetric methods are widely used in a large variety of fields, such as electrodeposition [3], batteries [4-7], fuel cells [8,9], supercapacitors [10, 11], electrochemical advanced oxidation [12-14], electrochemical sensors [15-18], ionic liquids [19-22] and surfactants [23], amongst many others.

Voltammetric techniques are electrochemical methods in which a potential, generally time-dependent, is applied to a working electrode, while the current flowing through the electrochemical cell is monitored [1]. These techniques are classified as active techniques, in opposition to passive techniques, since the applied potential modifies the concentration of electroactive species at the electrode surface and its surroundings. The representation of the current versus the applied potential is known as voltammogram, and is the voltammetric equivalent to a spectrum in spectroscopy: it provides both, qualitative and quantitative information on the system under study. Depending on the pattern of the applied potential with time, several types of voltammetries are defined. The most important ones are linear sweep voltammetry, staircase voltammetry, square wave voltammetry and cyclic voltammetry.

In cyclic voltammetries, the applied electrode potential is ramped linearly versus time. Unlike linear sweep voltammetries, in cyclic voltammetries, once the stop potential is reached, the applied potential is ramped in the opposite direction until the initial potential is reached. These cycles of potential ramps may be repeated as many times as required. The representation of the current versus the applied potential is known as cyclic voltammogram (CV). Nowadays, this electrochemical technique has become an important and widely used electroanalytical technique in many chemistry-related areas. It has been used to study the kinetics of redox processes [20], to study the stability of the reaction products [24], to determine the electron transfer kinetics [25] and to study the reversibility of redox reactions [26]. Furthermore, cyclic voltammetry has also been used to determine the formal reduction potential of an analyte [3], the ionic transport properties [19] and the diffusion coefficient of a given electroactive species [27]. 
Another common use of cyclic voltammetry is the identification of the intermediates of a given redox process [28] and the investigation of redox mechanisms [29].

In many cases, when a cyclic voltammetry measurement is performed, the system tends to a stationary state. It should be noted that, from a mathematical point of view, the stationary state concept is slightly different from the steady state one. On the one hand, in a steady state, the system variables are constant with time (i.e. $\neq f(t)$ ). On the other hand, in a stationary state, the system variables may present temporal variations (i.e. = $f(t)$ ), but their variation patterns repeat themselves in time. According to these definitions, a steady state is a particular case of stationary state, but not all stationary states are steady states. Since in the cyclic voltammetry context both signals (potential and current) are non-constant signals, the system variables change in time, and therefore, no proper steady state is reached during a CV measurement. Conversely, in a $\mathrm{CV}$ measurement the system reaches a stationary state: after a certain time, the signals display a cycle that repeats itself cyclically over time. The system is considered to be in a stationary state, when one cycle overlaps with the previous one. The limit cycle concept arises in this context: the limit cycle corresponds with the voltammogram to which the system tends. Once it reaches the limit cycle, the voltammogram does not change further if additional cycles are measured. Both, the convergence to a limit cycle and the non-convergence to a limit cycle, are highly informative. For instance, the fact that the system's voltammogram does not converge to a limit cycle, indicates that the system is not stable (v.g. electrode dissolution, concentration changes, etc...). For this reason, one of the first things to do in the preliminary analysis of cyclic voltammetry experimental results, is to assess whether the system converges to a limit cycle (and if it has reached the aforementioned limit cycle); or whether the system displays a time drift, and therefore does not converge to a limit cycle.

The common practice for assessing the voltammogram convergence is to perform a multicycle cyclic voltammetry (i.e. measure several cycles), and compare visually the sequential cycles in order to see if there are significant changes from one cycle to the following one. The main problem of visual comparison is its limited accuracy and its dependence on the analyst's subjectivity. In general, it is always preferable to use a quantitative method that allows to objectively assess the convergence for a given tolerance (i.e. numeric criterion). To the best of the author's knowledge, in literature there is no quantitative convergence assessment method for cyclic voltammetries.

This work tries to fill the mentioned gap in literature. Its goal is to develop an algorithm for quantitatively assessing the convergence of experimental cyclic voltammograms. The aforementioned algorithm will allow to decide whether the system's voltammogram has converged or not to its limit cycle, for a given numeric (i.e. objective) tolerance. In this work, the developed algorithm will be validated experimentally, by 
measuring the cyclic voltammograms of different experimental electrochemical systems and assessing their convergence.

\section{Convergence algorithm}

\subsection{Problem Statement}

As stated in the introduction section, in a cyclic voltammetry measurement, the potential is ramped linearly while the current is measured. The parameters that define a cyclic voltammetry are the initial potential, the final potential, the lower vertex potential, the upper vertex potential, the step potential (i.e. number of applied potentials), the scan rate and the number of cycles. Additionally, in some cases, cut-offs are considered: for instance, for only measuring the anodic or the cathodic branches (i.e. electrodes that can only withstand one type of polarization); or for limiting the magnitude of the current (i.e. limit the uncompensated resistance effect). Two types of cyclic voltammetries can be identified at this point:

- Type I CV: The list of applied potentials is the same from cycle to cycle. This is the common case when no cut-offs are considered. The list of applied potentials is defined by the vertex potentials and the number of applied potentials, and that does not change from one cycle to the following one.

- Type II CV: The list of applied potentials changes from cycle to cycle. This is usual when cut-offs are considered. For instance, if a high current cut-off is considered, since the cut-off may be reached at different potentials for each cycle, the effective upper vertex potential changes from cycle to cycle; and therefore, the applied potential list also changes from cycle to cycle.

Type I CV can be seen as a particular case of type II CV, since the latter is a more general case. Since this work intends to develop a general and versatile algorithm, it has to consider both cases. For this reason, the algorithm will be developed considering that the applied potential list can vary from one cycle to other.

Mathematically a voltammogram can be thought as a matrix of 2 columns: the first one contains the applied potentials, and the second one contains the measured currents. The matrix will have $N$ rows, where $N$ is the total number of applied potentials. This matrix contains $m N_{i} \times 2$ submatrices, where $m$ is the number of measured cycles and $N_{i}$ is the number of applied potentials during the $i$-th cycle. Logically:

$$
N=\sum_{i=1}^{m} N_{i}
$$


The following nomenclature will be used in this work:

$$
\boldsymbol{\Psi}=\left(\begin{array}{c}
\boldsymbol{\Psi}_{\mathbf{1}} \\
\boldsymbol{\Psi}_{2} \\
\vdots \\
\boldsymbol{\Psi}_{\boldsymbol{m}}
\end{array}\right)_{N \times 2}
$$

Where:

$$
\boldsymbol{\Psi}_{i}=\left(\begin{array}{cc}
E_{i, 1} & I_{i, 1} \\
E_{i, 2} & I_{i, 2} \\
\vdots & \vdots \\
E_{i, N_{i}} & I_{i, N_{i}}
\end{array}\right)_{N_{i} \times 2}
$$

$\Psi$ denotes the voltammogram matrix, while $\boldsymbol{\Psi}_{i}$ is the submatrix associated to the $i$-th cycle. $E_{i, k}$ denotes the $k$-th applied potential of the $i$-th cycle, and $I_{i, k}$ is the corresponding measured current. In cyclic voltammetry, each cycle is divided in two subcycles: an ascending subcycle, in which the voltage is swept in increasing order; and a descending subcycle, in which the voltage is swept in decreasing order. Therefore, the submatrix associated to the $i$-th cycle can be divided in two submatrices:

$$
\boldsymbol{\Psi}_{i}=\left(\begin{array}{c}
\boldsymbol{\Psi}_{i}^{\uparrow} \\
\boldsymbol{\Psi}_{i}^{\downarrow}
\end{array}\right)_{N_{i} \times 2}
$$

Where $\boldsymbol{\Psi}_{i}^{\uparrow}$ denotes the submatrix associated to the ascending subcycle of the $i$-th cycle, and $\boldsymbol{\Psi}_{i}^{\downarrow}$ represents the submatrix associated to the descending subcycle of the $i$-th cycle. In the above expression, it has been assumed that the ascending subcycle was performed before the descending one (i.e. the potential was first swept in increasing order, and then, in decreasing order). In case that the order of the subcycles was the opposite, the order of the submatrices would be reversed in equation (4). Following the proposed nomenclature, $E_{i, k}^{\uparrow}$ denotes the $k$-th applied potential of the ascending subcycle of the $i$-th cycle, and $I_{i, k}^{\uparrow}$ is the corresponding measured current. Analogously, $E_{i, k}^{\downarrow}$ and $I_{i, k}^{\downarrow}$ refer to the descending subcycle of the $i$-th cycle. In the general case, each subcycle may have a different number of applied potentials, $N_{i}^{\uparrow}$ and $N_{i}^{\downarrow}$, where $N_{i}=$ $N_{i}^{\uparrow}+N_{i}^{\downarrow}$.

The goal of the algorithm is to compare the different cycles of an experimental cyclic voltammogram, in order to evaluate their convergence. This comparison will be done point by point (i.e. applied potential by applied potential), and then, the information of all the points will be summed up by general convergence indicators. 


\subsection{Common potential range corrector}

In the general case (i.e. Type II CV), since the list of applied potentials changes from one cycle to another, the point-by-point comparison cannot be done directly. Before being able to perform it, the experimental data have to be transformed in order to refer them to a common potential list. For this reason, the first routine of the convergence assessment algorithm is the common potential range corrector (CPRC). The aforementioned routine takes as input 2 cycles of the experimental CV, which correspond to 2 submatrices, $\boldsymbol{\Psi}_{i}$ and $\boldsymbol{\Psi}_{j}$, of the $\mathrm{CV}$ matrix; and returns the corresponding transformed submatrices referred to a common list of applied potentials, $\left[\widetilde{\mathbf{\Psi}}_{i} ; \widetilde{\boldsymbol{\Psi}}_{j}\right]_{i, j}$ :

$$
\left[\left(\begin{array}{c}
\widetilde{\boldsymbol{\Psi}}_{i}^{\uparrow} \\
\widetilde{\boldsymbol{\Psi}}_{i}^{\downarrow}
\end{array}\right) ;\left(\begin{array}{c}
\widetilde{\boldsymbol{\Psi}}_{j}^{\uparrow} \\
\widetilde{\boldsymbol{\Psi}}_{j}^{\downarrow}
\end{array}\right)\right]_{i, j}=C P R C\left(\left(\begin{array}{c}
\boldsymbol{\Psi}_{i}^{\uparrow} \\
\boldsymbol{\Psi}_{i}^{\downarrow}
\end{array}\right) ;\left(\begin{array}{c}
\boldsymbol{\Psi}_{j}^{\uparrow} \\
\boldsymbol{\Psi}_{j}^{\downarrow}
\end{array}\right)\right)
$$

The output of the CPRC routine is a data structure that contains the two transformed submatrices. These transformed submatrices are included in a data structure since they do not have any sense on their own due to the fact that the transformation depends on both original submatrices. For instance, considering the following two transformed data structures, $\left[\widetilde{\boldsymbol{\Psi}}_{1} ; \widetilde{\boldsymbol{\Psi}}_{40}\right]_{1,40}$ and $\left[\widetilde{\boldsymbol{\Psi}}_{1} ; \widetilde{\boldsymbol{\Psi}}_{50}\right]_{1,50}$ : the transformed submatrix associated to the first cycle, $\widetilde{\boldsymbol{\Psi}}_{1}$, changes if the transformation is done with respect to the $40^{\text {th }}$ cycle or with respect to the $50^{\text {th }}$ cycle. For this reason, $\widetilde{\boldsymbol{\Psi}}_{1}$ does not have any sense on its own, and has to be accompanied by the second submatrix used for performing the transformation.

The first subroutine of the CPRC routine is the common potential list builder (CPLB) subroutine. This subroutine constructs the common potential list, which will be the first column of both transformed submatrices. This subroutine determines the overlap between the list of applied potentials of both considered cycles, and then, divides the aforementioned overlap in equally spaced potential intervals in order to obtain a new list of potentials that will be selected as the common potential list. In order to achieve this, the first step is to determine the overlap between both lists of applied potentials. Considering the transformation $\left[\widetilde{\Psi}_{i} ; \widetilde{\Psi}_{j}\right]_{i, j}$, the lower limit of the overlap can be determined using the following expressions:

$$
\begin{aligned}
& \breve{E}_{i, j}^{\uparrow}=\max \left\{\min _{k} E_{i, k}^{\uparrow} ; \min _{k} E_{j, k}^{\uparrow}\right\} \\
& \breve{E}_{i, j}^{\downarrow}=\max \left\{\min _{k} E_{i, k}^{\downarrow} ; \min _{k} E_{j, k}^{\downarrow}\right\}
\end{aligned}
$$


Analogously, the higher limit of the overlap is given by:

$$
\begin{aligned}
& \hat{E}_{i, j}^{\uparrow}=\min \left\{\max _{k} E_{i, k}^{\uparrow} ; \max _{k} E_{j, k}^{\uparrow}\right\} \\
& \hat{E}_{i, j}^{\downarrow}=\min \left\{\max _{k} E_{i, k}^{\downarrow} ; \max _{k} E_{j, k}^{\downarrow}\right\}
\end{aligned}
$$

In case that the overlap is an empty set (i.e. no overlap), $\widehat{E}_{i, j}<\breve{E}_{i, j}$; otherwise, $\widehat{E}_{i, j} \geq$ $\breve{E}_{i, j}$. These conditions can be used in order to detect if the applied potential lists overlap or not. Since convergence requires to be in the same potential range, in case $\widehat{E}_{i, j}<\breve{E}_{i, j}$, the CPRC routine stops and returns a "no overlap" error.

In case that $\widehat{E}_{i, j} \geq \breve{E}_{i, j}$, the next step of the CPLB subroutine is to divide in equally spaced intervals the overlapped potential range, $\left[\breve{E}_{i, j} ; \widehat{E}_{i, j}\right]$. In order to achieve this, first, the potential step has to be determined. In this work, the maximum number of points criterion was selected. According to this criterion, the number of points is given by:

$$
\begin{aligned}
& N_{i, j}^{\uparrow}=\max \left\{N_{i}^{\uparrow} ; N_{j}^{\uparrow}\right\} \\
& N_{i, j}^{\downarrow}=\max \left\{N_{i}^{\downarrow} ; N_{j}^{\downarrow}\right\}
\end{aligned}
$$

Once the number of points has been determined, the potential step follows immediately:

$$
\begin{aligned}
\Delta E_{i, j}^{\uparrow} & =\frac{\hat{E}_{i, j}^{\uparrow}-\breve{E}_{i, j}^{\uparrow}}{N_{i, j}^{\uparrow}} \\
\Delta E_{i, j}^{\downarrow} & =\frac{\hat{E}_{i, j}^{\downarrow}-\breve{E}_{i, j}^{\downarrow}}{N_{i, j}^{\downarrow}}
\end{aligned}
$$

Knowing the potential overlap and the potential step, the common list of applied potentials (i.e. output of the CPLB subroutine) can be easily built:

$$
\begin{aligned}
& \tilde{E}_{i, k}^{\uparrow}=\tilde{E}_{j, k}^{\uparrow}=\check{E}_{i, j}^{\uparrow}+(k-1) \cdot \Delta E_{i, j}^{\uparrow} \\
& \tilde{E}_{i, k}^{\downarrow}=\tilde{E}_{j, k}^{\downarrow}=\check{E}_{i, j}^{\downarrow}+(k-1) \cdot \Delta E_{i, j}^{\downarrow}
\end{aligned}
$$

With $k \in\left\{1 ; 2 ; \cdots ; N_{i, j}^{\uparrow / \downarrow}+1\right\}$. The above expressions define the first column of both transformed submatrices of the data structure $\left[\widetilde{\boldsymbol{\Psi}}_{i} ; \widetilde{\boldsymbol{\Psi}}_{j}\right]_{i, j}$. 
The second subroutine of the CPRC routine is the current linear interpolator (CLI) subroutine. This subroutine calculates the second column of the transformed submatrices, the transformed currents. In order to achieve this, the routine interpolates the measured currents (i.e. second column of the original submatrices) in order to obtain the corresponding current value at each potential of the common list built by the CPLB subroutine. Different types of interpolation methods (linear interpolation, cubic spline interpolation, piecewise cubic Hermite interpolation and barycentric Lagrange interpolation) were considered in a preliminary study: the obtained results were nearly identical despite the used interpolation method. This is due to the fact that the potential steps used in cyclic voltammetry are in general relatively small, and thus, the interpolation method has little effect on the interpolated values. Since all the interpolation methods present similar performances, the selection was done based on their computational cost. In order to minimize the computational cost of the convergence assessment algorithm, the simplest interpolation method from a computational point of view was selected: the linear interpolation method was used for implementing the CLI subroutine. Considering the transformation $\left[\widetilde{\Psi}_{i} ; \widetilde{\Psi}_{j}\right]_{i, j}$, the output of the $\mathrm{CLI}$ subroutine (i.e. the second column of the transformed submatrices) is given for the first submatrix by:

$$
\begin{aligned}
& \tilde{I}_{i, k}^{\uparrow}=I_{i, \alpha_{i, k}^{\uparrow}}^{\uparrow}+\left(\tilde{E}_{i, k}^{\uparrow}-E_{i, \alpha_{i, k}^{\uparrow}}^{\uparrow}\right) \cdot \frac{I_{i, \alpha_{i, k}^{\uparrow}}^{\uparrow}-I_{i, \beta_{i, k}^{\uparrow}}^{\uparrow}}{E_{i, \alpha_{i, k}^{\uparrow}}^{\uparrow}-E_{i, \beta_{i, k}^{\uparrow}}^{\uparrow}} \\
& \tilde{I}_{i, k}^{\downarrow}=I_{i, \alpha_{i, k}^{\downarrow}}^{\downarrow}+\left(\tilde{E}_{i, k}^{\downarrow}-E_{i, \alpha_{i, k}^{\downarrow}}^{\downarrow}\right) \cdot \frac{I_{i, \alpha_{i, k}^{\downarrow}}^{\downarrow}-I_{i, \beta_{i, k}^{\downarrow}}^{\downarrow}}{E_{i, \alpha_{i, k}^{\downarrow}}^{\downarrow}-E_{i, \beta_{i, k}^{\downarrow}}^{\downarrow}}
\end{aligned}
$$

And for the second submatrix:

$$
\begin{aligned}
& \tilde{I}_{j, k}^{\uparrow}=I_{j, \alpha_{j, k}^{\uparrow}}^{\uparrow}+\left(\tilde{E}_{j, k}^{\uparrow}-E_{j, \alpha_{j, k}^{\uparrow}}^{\uparrow}\right) \cdot \frac{I_{j, \alpha_{j, k}^{\uparrow}}^{\uparrow}-I_{j, \beta_{j, k}^{\uparrow}}^{\uparrow}}{E_{j, \alpha_{j, k}^{\uparrow}}^{\uparrow}-E_{j, \beta_{j, k}^{\uparrow}}^{\uparrow}} \\
& \tilde{I}_{j, k}^{\downarrow}=I_{j, \alpha_{j, k}^{\downarrow}}^{\downarrow}+\left(\tilde{E}_{j, k}^{\downarrow}-E_{j, \alpha_{j, k}^{\downarrow}}^{\downarrow}\right) \cdot \frac{I_{j, \alpha_{j, k}^{\downarrow}}^{\downarrow}-I_{j, \beta_{j, k}^{\downarrow}}^{\downarrow}}{E_{j, \alpha}^{\downarrow}-E_{j, k}^{\downarrow}}
\end{aligned}
$$

In the above expressions, $\alpha_{k}$ denotes the largest index for which $E_{\alpha_{k}}$ is lower than $\tilde{E}_{k}$; and $\beta_{k}$ denotes the lowest index for which $E_{\beta_{k}}$ is higher than $\tilde{E}_{k}$.

Finally, the output of the CPRC routine is built by combining properly the outputs of the CPLB and the CLI subroutines: 


$$
\operatorname{CPRC}\left(\boldsymbol{\Psi}_{i} ; \boldsymbol{\Psi}_{j}\right)=\left[\left(\begin{array}{cc}
\tilde{E}_{i, 1}^{\uparrow} & \tilde{I}_{i, 1}^{\uparrow} \\
\tilde{E}_{i, 2}^{\uparrow} & \tilde{I}_{i, 2}^{\uparrow} \\
\vdots & \vdots \\
\tilde{E}_{i, N_{i, j}^{\uparrow}}^{\uparrow} & \tilde{I}_{i, N_{i, j}^{\uparrow}}^{\uparrow} \\
\tilde{E}_{i, 1}^{\downarrow} & \tilde{I}_{i, 1}^{\downarrow} \\
\tilde{E}_{i, 2}^{\downarrow} & \tilde{I}_{i, 2}^{\downarrow} \\
\vdots & \vdots \\
\tilde{E}_{i, N_{i, j}^{\downarrow}}^{\downarrow} & \tilde{I}_{i, N_{i, j}^{\downarrow}}^{\downarrow}
\end{array}\right) ;\left(\begin{array}{cc}
\tilde{E}_{j, 1}^{\uparrow} & \tilde{I}_{j, 1}^{\uparrow} \\
\tilde{E}_{j, 2}^{\uparrow} & \tilde{I}_{j, 2}^{\uparrow} \\
\vdots & \vdots \\
\tilde{E}_{j, N_{i, j}^{\uparrow}}^{\uparrow} & \tilde{I}_{j, N_{i, j}^{\uparrow}}^{\uparrow} \\
\tilde{E}_{j, 1}^{\downarrow} & \tilde{I}_{j, 1}^{\downarrow} \\
\tilde{E}_{j, 2}^{\downarrow} & \tilde{I}_{j, 2}^{\downarrow} \\
\vdots & \vdots \\
\tilde{E}_{j, N_{i, j}}^{\downarrow} & \tilde{I}_{j, N_{i, j}^{\downarrow}}^{\downarrow}
\end{array}\right]_{i, j}\right.
$$

As a final remark, it should be noted that in the case of a type I CV (i.e. the list of applied potentials does not change form cycle to cycle), the submatrices of the CV matrix are invariant with respect to the CPRC transformation: the output of the CPRC routine is exactly its input, in the case of type I CVs. This is exactly what is expected, since in type I CVs, the potential lists are already the same from one submatrix to another, and therefore the CPRC transformation is immediate.

\subsection{Comparator}

Once both submatrices that have to be compared, have been referred to a common potential list, they can be compared point-by-point in order to quantify how different they are. The comparator routine performs this task. It takes as input the data structure built by the CPRC routine, and returns a single scalar value that quantifies the difference between the two compared cycles of the cyclic voltammetry. The comparator routine is divided in two subroutines: the vectorial relative comparator (VRC) subroutine and the error vector synthesizer (EVS) subroutine.

On the one hand, the VCR subroutine takes as input the CPRC data structure, and returns a data structure composed of two error vectors, one associated to the ascending subcycle and the other one associated to the descending subcycle:

$$
\operatorname{VRC}\left(\left[\widetilde{\mathbf{\Psi}}_{i} ; \widetilde{\mathbf{\Psi}}_{j}\right]_{i, j}\right)=\left[\vec{\varepsilon}_{i, j}^{\uparrow} ; \vec{\varepsilon}_{i, j}^{\downarrow}\right]=\left[\left(\begin{array}{c}
\varepsilon_{i, j, 1}^{\uparrow} \\
\varepsilon_{i, j, 2}^{\uparrow} \\
\vdots \\
\varepsilon_{i, j, N_{i, j}^{\uparrow}}^{\uparrow}
\end{array}\right) ;\left(\begin{array}{c}
\varepsilon_{i, j, 1}^{\downarrow} \\
\varepsilon_{i, j, 2}^{\downarrow} \\
\vdots \\
\varepsilon_{i, j, N_{i, j}^{\downarrow}}^{\downarrow}
\end{array}\right)\right]
$$

The $k$-th entry of the error vector, $\varepsilon_{i, j, k}$, quantifies the difference between the transformed current of cycle $i$ and the transformed current of cycle $j$, for the $k$-th potential of the common potential list. In order to make the comparison independent of the order of magnitude of the currents, relative errors taking the second cycle as the 
reference were used in this work. Furthermore, it is incoherent to build an algorithm that works with a current resolution higher than the current resolution of the experimental measurement of the $\mathrm{CV}$. For this reason, the algorithm only calculates the error if the current difference is relevant (i.e. higher than the resolution of the experimental measurement); and considers that the relative error is 0 in the other case. This idea was implemented using a thresholder:

$$
\begin{aligned}
\varepsilon_{i, j, k}^{\uparrow} & = \begin{cases}0 & \text { if }\left|\tilde{I}_{i, k}^{\uparrow}-\tilde{I}_{j, k}^{\uparrow}\right| \leq \delta \\
\left|\frac{\tilde{I}_{i, k}^{\uparrow}-\tilde{I}_{j, k}^{\uparrow}}{\tilde{I}_{j, k}^{\uparrow}}\right| \cdot 100 & \text { if }\left|\tilde{I}_{i, k}^{\uparrow}-\tilde{I}_{j, k}^{\uparrow}\right|>\delta\end{cases} \\
\varepsilon_{i, j, k}^{\downarrow} & = \begin{cases}0 & \text { if }\left|\tilde{I}_{i, k}^{\downarrow}-\tilde{I}_{j, k}^{\downarrow}\right| \leq \delta \\
\left|\frac{\tilde{I}_{i, k}^{\downarrow}-\tilde{I}_{j, k}^{\downarrow}}{\tilde{I}_{j, k}^{\downarrow}}\right| \cdot 100 & \text { if }\left|\tilde{I}_{i, k}^{\downarrow}-\tilde{I}_{j, k}^{\downarrow}\right|>\delta\end{cases}
\end{aligned}
$$

With $k \in\left\{1 ; 2 ; \cdots ; N_{i, j}^{\uparrow / \downarrow}\right\} . \delta$ denotes the current threshold that defines the level of significance from which the algorithm considers that the current difference is relevant. As a rule of thumb, authors recommend to use the significance level of the experimental measurement (i.e. the current resolution of the selected measurement range) as threshold.

On the other hand, the EVS subroutine takes as input the error vector data structure built by the VCR subroutine, combines the different errors contained in the error vectors, and returns a single scalar value that is representative of the error vectors:

$$
\sigma_{i, j}=\operatorname{EVS}\left(\left[\vec{\varepsilon}_{i, j}^{\uparrow} ; \vec{\varepsilon}_{i, j}^{\downarrow}\right]\right)=\operatorname{mean}\left\{\operatorname{med}_{k} \varepsilon_{i, j, k}^{\uparrow} ; \operatorname{med}_{k} \varepsilon_{i, j, k}^{\downarrow}\right\}
$$

Where med denotes the median operator.

\subsection{Convergence indicators}

Two convergence indicators were considered in this work: a cycle-to-cycle convergence indicator, $\theta$; and a long term convergence indicator, $\Phi$. The cycle-to-cycle convergence indicator compares each cycle with the cycle immediately before; whereas, the long term convergence indicator compares all the cycles with the last cycle. These indicators can be calculated using the routines defined in previous subsections.

On the one hand, for $i \in\{2 ; 3 ; \cdots ; m\}$ :

$$
\theta_{i}=\operatorname{EVS}\left(\operatorname{VRC}\left(\operatorname{CPRC}\left(\boldsymbol{\Psi}_{i-1} ; \boldsymbol{\Psi}_{i}\right)\right)\right)
$$


And on the other hand, for $i \in\{1 ; 2 ; \cdots ; m-1\}$ :

$$
\Phi_{i}=\operatorname{EVS}\left(\operatorname{VRC}\left(\operatorname{CPRC}\left(\boldsymbol{\Psi}_{i} ; \boldsymbol{\Psi}_{m}\right)\right)\right)
$$

\subsection{Algorithm summary and practical implementation}

Figure 1 shows the block diagram of the proposed convergence assessment algorithm. This algorithm can be easily automated in any programing language. In this work, the algorithm was fully implemented in Labview $\AA$ : the Labview $\AA$ program directly takes as input the .txt output of NOVA®, the software used here to control the potentiostat; and returns the convergence indicators.

Figure 2 shows the required computational time for executing the developed

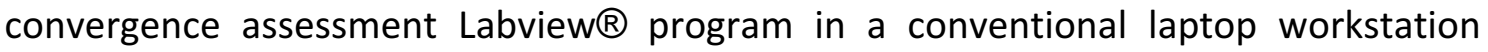
(Intel® Core $囚 \mathrm{i} 5-2410 \mathrm{M}$ at $2.30 \mathrm{GHz}, 4 \mathrm{~Gb}$ RAM and 64-bit Windows $\circledR$ 10) for different cycle sizes (figure 2.a) and different number of cycles of a given size (figure 2.b). The inset in figure 2.a corresponds with a zoom of figure 2.a at low cycle sizes. Theses execution times were obtained executing the Labview ${ }^{\circledR}$ program on its own, with no background programs, except the operating system itself and its utilities. On the one side, it can be observed that for few applied potentials, the execution time per cycle remains constant; whereas, for cycles of 300 applied potentials or more, the execution time per cycle increases linearly with the number of applied potentials. On the other side, it can be observed that the execution time increases linearly with the number of cycles of a given size. These two observations can be summed up by:

$$
t=0.00096 \cdot \sum_{i=1}^{m} N_{i}
$$

Where $t$ denotes the execution time.

These results indicate that the developed program exhibits a linear growth rate, $\mathcal{O}(n)$. The proportionality constant, $\lambda$, is $0.96 \mu \mathrm{s} \cdot$ point $^{-1}$. Figure 3 displays the computation time breakdown in the different subroutines of the algorithm. It can be observed that the subroutine that consumes the greatest part of the computation time is the CLI subroutine, which represents a $69 \%$ of the total computational time. The CPRC routine $(\mathrm{CPLB}+\mathrm{CLI})$ represents three quarters of the total computational time. Consequently, for type I CVs, the execution time is reduced by $75 \%$. These execution time results show that even when it is run on a conventional personal laptop, the required execution times are low enough in order to allow executing the algorithm in real time during the cyclic voltammetry measurement. This is one of the main strengths of the developed 
algorithm: it allows to automate the convergence assessment in real time during the measurement.

However, there is a maximum scan rate that can be handled in real time by the algorithm: scan rates above this maximum scan rate cannot be analysed in real time by the algorithm (i.e. the measurement is faster than the convergence assessment algorithm). This maximum scan rate can be estimated using the following expression:

$$
v_{\max } \sim \frac{\Delta E}{\lambda}
$$

Where $v_{\text {max }}$ denotes the maximum scan rate that can be handled in real time by the algorithm, $\Delta E$ is the step size, and $\lambda$ is the time required for the algorithm to analyse a new point. $\lambda$ depends on the particular implementation of the algorithm (i.e. some implementations are more efficient from a computational point of view) and on the computer the program is run on. As seen before, for the implementation of the algorithm considered in this work, and the computer on which it was run, $\lambda=0.96 \mu \mathrm{s}$. point $^{-1}$. Therefore, for a typical potential step of $1 \mathrm{mV} \cdot$ point $^{-1}$, the maximum scan rate is in the $k V \cdot s^{-1}$ order. If faster scan rates are desired (i.e. fast-scan CVs use scan rates in the $M V \cdot s^{-1}$ order), two options are available: the step size can be increased, or the algorithm speed can be increased (i.e. lower $\lambda$ ) by implementing the algorithm in a more efficient way or by using a faster computer.

\section{Experimental work}

In order to experimentally validate the algorithm presented in this work, two electrochemical systems were considered: one for obtaining type I CVs, and the other one for obtaining type II CVs. Both systems consisted in a conventional 3-electrode thermostatted electrochemical cell. A commercial Pt ring electrode (Crison® ${ }^{8267)}$ was used as the counter-electrode, and a commercial $\mathrm{Ag} / \mathrm{AgCl}(3 \mathrm{M} \mathrm{KCl})$ electrode (Crison $\circledR$ 5240) was used as the reference electrode. All the experiments were done at $30^{\circ} \mathrm{C}$, using a $0.1 \mathrm{M} \mathrm{Na}_{2} \mathrm{SO}_{4}$ (Panreac®) electrolyte. In the first system, the working electrode was a mirror polished Pt electrode; whereas in the second system, the working electrode was one of the $\mathrm{Sb}$-doped $\mathrm{SnO}_{2}$ ceramic electrodes presented in the work of Mora-Gómez and co-workers [30]. The particularity of these ceramic electrodes, from a cyclic voltammetry perspective, is that they cannot withstand cathodic polarization, since this type of polarization would irreversibly reduce the tin oxide, producing permanent electrode damage [31].

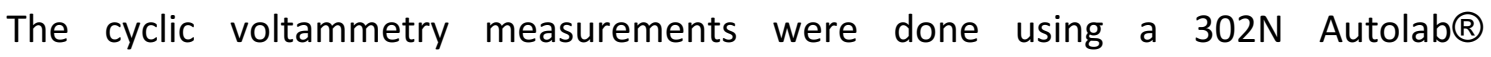
potentiostat/galvanostat with FRA module, controlled with NOVA® software. On the 
one side, for the first system, 100 cycles were measured at a scan rate of $10 \mathrm{mV} \cdot \mathrm{s}^{-1}$. The sweep was done first in ascending sense, and then, in descending sense, with an upper potential vertex of $+1.4 \mathrm{~V}$ and a lower potential vertex of $-0.9 \mathrm{~V}$. No cut-offs were considered for the first system. The CVs obtained with these measurement parameters are type I CVs. On the other side, for the second system, 100 cycles were measured at a scan rate of $10 \mathrm{mV} \cdot \mathrm{s}^{-1}$. The sweep was started at $\mathrm{OCP}$, and it was done first in ascending sense, and then, in descending sense, with an upper potential vertex of $+4.0 \mathrm{~V}$ and a lower potential vertex of $-4.0 \mathrm{~V}$. Since the cathodic branch has to be avoided, a scan direction reversal cut-off was considered for $I<1 \cdot 10^{-8} A$ (i.e. only the anodic branch is measured). The CVs obtained with these measurement parameters are type II CVs.

A very big electrolyte volume was used in both systems in order to ensure that the bulk electrolyte concentration remained constant throughout the whole cyclic voltammetry measurement, avoiding in this way time drifts due to bulk concentration variations that would prevent the CV from converging to its limit cycle. Moreover, before each experiment, the electrolyte was stirred vigorously for 20 minutes in order to ensure a complete homogenization of the electrolyte.

\section{$\underline{4 .}$ Experimental results and discussion}

\subsection{Type I CV}

Figure 4 presents some of the experimental cyclic voltammograms obtained for the system with the Pt working electrode. In the aforementioned figure, the sweep direction is shown by the arrows, and point $P_{0}$ marks the first measured point. It can be observed that the list of applied potentials (i.e. horizontal position of the different points) is the same in every cycle: it is a type I CV.

These experimental voltammograms display the typical peaks associated to a $\mathrm{Pt}$ electrode [32]: the oxygen evolution reaction $\left(\mathrm{H}_{2} \mathrm{O} \rightarrow \mathrm{O}_{2}\right)$, the hydrogen evolution reaction $\left(\mathrm{H}^{+} \rightarrow \mathrm{H}_{2}\right)$, the hydrogen dissolution reaction $\left(\mathrm{H}_{2} \rightarrow \mathrm{H}^{+}\right)$and the platinum oxide reduction reaction (PtO $\rightarrow P t$ ). As it can be clearly observed in figure 4 , the CV tends to its limits cycle: in the first cycles, the CV changes appreciably from cycle to cycle; whereas, in the last cycles, no relevant changes can be observed from cycle to cycle. For example, as it can be seen in figure 4.c, cycles 99 and 100 seem totally superimposable by visual comparison. This observation demonstrates the need of assessing the convergence of a CV before accepting and analysing further the experimental results of a cyclic voltammetry measurement. For instance, in this case, if the first cycle was accepted without assessing the convergence, a substantial error would be made with respect to the limit cycle, as it can be seen in figure 4.b. 
Figure 5 presents the obtained results when the developed convergence assessment algorithm was applied to the experimental data shown in figure 4. These results consist of the representation of the two considered convergence indicators (cycle-to-cycle convergence indicator and long term convergence indicator) versus the cycle number. As recommended in section 2.3 , the selection of the threshold for the convergence assessment algorithm was done based on the current range selected during the experimental measurement: $\delta=1 \mu A$.

The immediate observation that can be extracted from figure 5 , is that both indicators present a global decreasing trend with the cycle number. On the one hand, the decreasing trend of the cycle-to-cycle convergence indicator indicates that the "difference" between one cycle and the previous one, decreases as cycles follow each other. This is a necessary, but no sufficient, condition for CV convergence. On the other hand, the long term convergence indicator initially presents a clear decreasing trend, and then, it stabilizes around a very low value. On the one hand, the initial decreasing trend indicates that the "difference" between each of the first cycles and the last one, decreases as cycles follow each other. On the other hand, the quasi-stationary trend indicates that the "difference" between the corresponding cycles and the last one is approximately constant for all these cycles, and this difference is very low. Therefore, the cycles associated to the quasi-stationary trend can be considered to be approximately equal to the last cycle. This is exactly the definition of CV convergence. The fact that the long term convergence indicator, $\Phi$, stabilizes around a very low value (i.e. nearly 0 ) indicates that the CV has converged to its limit cycle. Moreover, the value around which the indicator stabilizes, quantifies the measurement noise (i.e. how much the CV changes just because of the measurement error).

In this work, an arbitrary convergence criterion of $1 \%$ was selected. According to this criterion, both indicators converge simultaneously at cycle 65 . Therefore, all the cycles from cycle 65 to cycle 100 can be considered as the same cycle (i.e. the limit cycle). Since from a practical point of view, they are the same cycle, they can be combined in order to reduce the measurement error.

\subsection{Type II CV}

Figure 6 presents some of the experimental cyclic voltammograms obtained for the system with the Sb-doped $\mathrm{SnO}_{2}$ ceramic working electrode. As in figure 4, the sweep direction is displayed by arrows, and point $P_{0}$ corresponds with the first measured point. In this case, it is clear that the list of applied potentials (i.e. horizontal position of the different points) changes from cycle to cycle. For instance, as it can be seen in figure 6.b, the list of applied potentials during the first cycle ranges from $1 \mathrm{~V} \mathrm{vs} \mathrm{Ag} / \mathrm{AgCl}$ to 
$4 \mathrm{~V}$ vs $\mathrm{Ag} / \mathrm{AgCl}$; whereas, the list of applied potentials during the last cycle ranges from $1.7 \mathrm{~V} v s \mathrm{Ag} / \mathrm{AgCl}$ to $4 \mathrm{~V} v s \mathrm{Ag} / \mathrm{AgCl}$. This is a clear example of type II CV.

These experimental voltammograms only present anodic domains, and not cathodic domains. This is the result of the inclusion of the reversal cut-off condition, that as explained in section 3, was considered in order to prevent the cathodic polarization of the ceramic electrode that would irreversibly damage it. These voltammograms display the typical anodic branch related to the oxygen evolution reaction $\left(\mathrm{H}_{2} \mathrm{O} \rightarrow \mathrm{O}_{2}\right)$. Figure 6 demonstrates clearly that the CV tends to its limit cycle: the CV changes appreciably from cycle to cycle for the first cycles, and then, it does not change further for the last cycles. For instance, as shown in figure 6.c, cycles 99 and 100 are visually superimposable. As in the Pt electrode case, these results demonstrate the need of assessing the CV convergence: in this case the error of the first cycle with respect to the limit cycle is even greater than in the Pt case, as it can be deduced from figure 6.b.

Figure 7 presents the convergence assessment results associated to the experimental data shown in figure 6 . These results were obtained using a $\delta=1 \mu A$ threshold, selected based on the current range used to perform the experimental measurements. As in the Pt case, for the ceramic electrode, both convergence indicators also present a global decreasing trend with the cycle number. On the one hand, as it can be observed in figure 7.a, the decreasing trend of the cycle-to-cycle convergence indicator indicates that the "difference" between one cycle and the previous one, decreases as cycles follow each other. On the other hand, as it can be observed in figure 7.b, the long term convergence indicator presents the same trend that it presented in the Pt electrode case, though in the ceramic electrode case the trend is much more marked: initially, $\Phi$ decreases with the cycle number, and then, it stabilizes around a nearly 0 value. As explained in section 4.1, this indicates that the CV has converged to its limit cycle.

Using the same convergence criterion than in section 4.1, the cycle-to-cycle indicator converges at cycle 23, whereas the long term indicator converges at cycle 50 . In this case, both convergence indicators do not converge simultaneously. This indicates that the "difference" between two consecutives cycles is little, but since the change is always in the same direction (not random as with measurement errors), there is a global time shift. This demonstrates the statement made in the section 4.1, according to which the convergence of the cycle-to-cycle indicator is a necessary, but not sufficient, condition for CV convergence. Furthermore, this justifies the need to consider a long term convergence indicator in the algorithm, and not only a cycle-to-cycle indicator. The final conclusion that can be extracted from figure 7 , is that the ceramic electrode CVs converge at cycle 50: all the cycles from cycle 50 to cycle 100 can be considered as the same cycle (i.e. the limit cycle), and can be combined in order to reduce the measurement error. 
Comparing the convergence studies of both systems, the first observation that can be extracted is that the ceramic electrode system converges to its limit cycle before the Pt electrode system: the first only requires 50 cycles to converge, while the later requires 65. Moreover, as stated in section 4.1, the value around which the long term convergence indicator stabilizes, quantifies the measurement noise. In the Pt electrode case, $\Phi$ stabilizes around $0.44 \%$; while in the ceramic electrode case, it stabilizes around $0.48 \%$. This suggests that the measurement noise level is similar in both systems. This could be expected since the same measurement equipment was used in both systems which, in addition, are quite similar (i.e. the only difference is the working electrode).

\section{$\underline{\text { 5. Conclusions }}$}

In conclusion, in this work an algorithm has been developed in order to assess the convergence of experimental cyclic voltammetry measurements to their limit cycle. The algorithm is based in two convergence indicators: a cycle-to-cycle convergence indicator and a long term convergence indicator. The algorithm was successfully validated experimentally using two systems, one that generated type I CVs and the other one that generated type II CVs. In both cases, the algorithm was able to determine whether the CV converged to its limit cycle, and when (i.e. at which cycle) it converged. Moreover, the algorithm is able to quantify the measurement noise (i.e. how much the CV changes just because of the measurement error).

Determining if the $\mathrm{CV}$ has converged is important in order to avoid errors due to accepting a CV which does not correspond with the limit cycle. Furthermore, determining when the $\mathrm{CV}$ converged, allows to combine all the cycles after convergence, reducing in this way the measurement error.

At this point, someone could argue that there is no need to use an algorithm for assessing the convergence of a CV, since it can be done using the traditional way (i.e. by visual inspection). The first advantage of using the developed algorithm with respect to the visual inspection method, is that the algorithm is an objective and quantitative method, whereas visual inspection is subjective: the algorithm allows to determine at which cycle convergence has been achieved according to a certain numeric convergence criterion, while this is much more difficult using the visual inspection method. However, this is only a minor advantage. The definitive advantage of the algorithm over the visual inspection method arises from its low computational cost. The execution time of the algorithm is low enough in order to execute it in real time during the cyclic voltammetry measurement. In this way, it can be used in order to decide when to stop cycling: rather than selecting the number of cycles as in traditional cyclic voltammetry, convergence criteria would be selected. Some of these criteria could be: limit in the convergence 
indicators and number of cycles below the limit, maximum number of cycles, etc.... Then, the measurement software would cycle until the convergence criteria are met. In this way, the measurement could be completely automated, and would not require the intervention of the operator at any point. This could be extremely useful in some cases, as overnight measurements.

It should be noted that the algorithm only assesses whether a CV tends to a limit cycle or not, and if it does, how many cycles are required in order to converge to the limit cycle. However, the algorithm does not give any clue about the causes that lead to the existence or the non-existence of a limit cycle in a particular system. The list of possible causes of the existence/non-existence of a limit cycle is quite large and includes surface reorganization, double layer formation, surface dissolution and edge effects, amongst others. It is the researcher who will have to propose possible hypothetic causes that explain why his particular system tends or not to a limit cycle, gaining in this manner interesting insights on the studied system from the convergence study results. 


\section{Nomenclature}

\section{Latin symbols}

E $\quad$ Electrode potential $(V)$

$E_{i, k} \quad k$-th applied potential of the $i$-th cycle $(V)$

$\tilde{E}_{i, k} \quad k$-th transformed potential of the $i$-th cycle $(V)$

$\breve{E} \quad$ Lower limit of the overlap of the applied potential lists $(V)$

$\hat{E} \quad$ Higher limit of the overlap of the applied potential lists $(V)$

I Current $(A)$

$I_{i, k} \quad k$-th measured current of the $i$-th cycle $(A)$

$\tilde{I}_{i, k} \quad k$-th transformed current of the $i$-th cycle $(A)$

$N \quad$ Total number of applied potentials (all cycles)

$N_{i} \quad$ Total number of applied potentials during the $i$-th cycle

$m \quad$ Number of cycles

\section{Greek symbols}

$\alpha_{k} \quad$ Largest index for which $E_{\alpha_{k}}$ is lower than $\tilde{E}_{k}$

$\beta_{k} \quad$ Lowest index for which $E_{\beta_{k}}$ is higher than $\tilde{E}_{k}$

$\Delta E \quad$ Potential step $(V)$

$\delta \quad$ Current threshold $(A)$

$\vec{\varepsilon}_{i, j} \quad$ Error vector associated with the comparison of the $i$-th cycle and the $j$-th cycle

$\theta \quad$ Cycle-to-cycle convergence indicator

$\Phi \quad$ Long term convergence indicator

$\Psi \quad$ Voltammogram matrix

$\Psi_{i} \quad$ Submatrix of the voltammogram matrix associated to the $i$-th cycle

$\left[\widetilde{\boldsymbol{\Psi}}_{i} ; \widetilde{\boldsymbol{\Psi}}_{j}\right]_{i, j} \quad$ Data structure containing the CPRC-transformed submatrices

\section{Superscripts}
$\uparrow \quad$ Related to the ascending subcycle
$\downarrow \quad$ Related to the descending subcycle

\section{Acronyms}

CLI "Current linear interpolator" subroutine

$C P L B \quad$ "Common potential list builder" subroutine

$C P R C \quad$ "Common potential range corrector" routine 
EVS "Error vector synthesizer" subroutine

VRC "Vectorial relative comparator" subroutine

\section{$\underline{\text { 7. Acknowledgments }}$}

The authors are very grateful to the Generalitat Valenciana (Vali+d postdoctoral grant APOSTD/2018/001), to the Ministerio de Economia y Competitividad (Project CTQ201565202-C2-1-R), to the European Regional Development Fund (FEDER) and to the European Social Fund, for their economic support.

\section{Bibliography}

[1] C.G. Zoski, Handbook of electrochemistry, Elsevier, Paris (2007).

[2] D.A. Skoog, D.M. West, F.J. Holler and S. Crouch, Fundamentals of analytical chemistry, Brook \& Cole, New York (2013).

[3] W. Sides, N. Kassouf and Q. Huang, J. Electrochem. Soc., 166, D77 (2019).

[4] S.S. Zhang, J. Chen and C. Wang, J. Electrochem. Soc., 166, A487 (2019).

[5] Z. Meng, Y. Huang, J. Li, R. Yang, X. Wang, Y. Guo, Z. Liu, M. Xi, W. Su and Lei Wang, J. Electrochem. Soc., 166, A546 (2019).

[6] S. Watanabe, D. Mori, S. Taminato, Y. Matsuda, O. Yamamoto, Y. Takeda and N. Imanishi, J. Electrochem. Soc., 166, A539 (2019).

[7] X. Zhou, T. Pu, G. Yang, W. Ma, B. Yang and Y. Dai, J. Electrochem. Soc., 166, A448 (2019).

[8] J.J. Giner-Sanz, E.M. Ortega and V. Pérez-Herranz, Fuel Cells, 15, 479 (2015).

[9] J.J. Giner-Sanz, E.M. Ortega and V. Pérez-Herranz, Int. J. Hydrogen Energ., 39, 13206 (2014).

[10] V. Naresh and S.K. Martha, J. Electrochem. Soc., 166, A551 (2019).

[11] T. Fan, P. Sun, J. Zhao, Z. Cui and G. Cui, J. Electrochem. Soc., 166, D37 (2019).

[12] L. Xu, Y. Wang, Q. Xu and H. Duan, J. Electrochem. Soc., 166, E69 (2019). 
[13] M.J. Sánchez-Rivera, J.J. Giner-Sanz, V. Pérez-Herranz, S. Mestre, Int. J. Appl. Ceram. Tec. doi.org/10.1111/ijac.13149.

[14] J.J. Giner-Sanz, M.J. Sánchez-Rivera, M. García-Gabaldón, E.M. Ortega, S. Mestre and V. Pérez-Herranz, ChemElectroChem doi.org/10.1002/celc.201801766.

[15] S. Dong, H. Cui, D. Zhang and M. Tong, J. Electrochem. Soc., 166, B193 (2019).

[16] J. Wu, Y. Zhu, K. Yan and J. Zhang, J. Electrochem. Soc., 166, H87 (2019).

[17] N.F. Atta, A. Galal, E.H. El-Ads and A.E. Galal, J. Electrochem. Soc., 166, B161 (2019).

[18] K. Ma, A. Sinha, X. Dang and H. Zhao, J. Electrochem. Soc., 166, B147 (2019).

[19] N.C. Osti, B. Dyatkin, A. Gallegos, D. Voneshen, J.K. Keum, K. Littrell, P. Zhang, S. Dai, J. Wu, Y. Gogotsi and E. Mamontov, J. Electrochem. Soc., 166, A507 (2019).

[20] L.F. Loguercio, C.F. de Matos, M.C. de Oliveira, G. Marin, S. Khan, J. Dupont, S. Ribeiro-Teixeira, N.M. Balzaretti, J.F. Leite-Santos and M.J. Leite-Santos, J. Electrochem. Soc., 166, H3188 (2019).

[21] S. Thomas, D. Kowalski, M. Molinari and J. Mallet, Electrochim. Acta, 265, 166 (2018).

[22] Q. Zhang, X. Liu, L. Yin, P. Chen, Y. Wang and T. Yan, Electrochim. Acta, 270, 352 (2018).

[23] B.N. Viada, A.V. Juárez, E.M.P. Gómez, M.A. Fernández, L.M. Yudi, Electrochim. Acta, 263, 499 (2018).

[24] E. Vijayakumar, Y.H. Yun, V.H.V. Quy, Y.H. Lee, S.H. Kang, K.S. Ahn and S.W. Lee, J. Electrochem. Soc., 166, D86 (2019).

[25] A.M. Kosswattaarachchi, L.E. VanGelder, O. Nachtigall, J.P. Hazelnis, W.W. Brennessel, E.M. Matson and T.R. Cook, J. Electrochem. Soc., 166, A464 (2019).

[26] B. Tang, J. Zhou, G. Fang, S. Guo, X. Guo, L. Shan, Y. Tang and S. Liang, J. Electrochem. Soc., 166, A480 (2019).

[27] Y. Li, Y. Zhang, J. Ma, L. Yang, X. Li, E. Zhao, S. Fan, G. Xu, S. Yang and C. Yang, J. Electrochem. Soc., 166, A410 (2019). 
[28] M. Li, Y. Li and Z. Wang, J. Electrochem. Soc., 166, D65 (2019).

[29] L. Du, W. Wu, C. Luo, D. Xu, H. Guo, R. Wang, T. Zhang, J. Wang and Y. Deng, J. Electrochem. Soc., 166, A423 (2019).

[30] J. Mora-Gomez, M. Garcia-Gabaldon, E.M. Ortega, M.J. Sánchez-Rivera, S. Mestre and V. Pérez-Herranz, Ceram. Int., 44, 2216 (2018).

[31] F. Montilla, E. Morallón, A. De Battisti and J.L. Vázquez, J Phys. Chem. B, 108, 5036 (2004).

[32] P. Daubinger, J. Kieninger, T. Unmüssig and G.A. Urban, Phys. Chem. Chem. Phys., 16, 8392 (2014). 


\section{LIST OF FIGURES:}

Figure 1. Convergence assessment algorithm block diagram. a.) Convergence assessment algorithm. b.) Common potential range corrector routine. c.) Comparator routine

Figure 2. Execution time of the convergence assessment algorithm. a.) Execution time as a function of the number of applied potentials. b.) Execution time as a function of the number of cycles of 600 applied potentials

Figure 3. Computation time breakdown

Figure 4. Experimental cyclic voltammograms measured for the Pt electrode (Type I CVs), at a scan rate of $10 \mathrm{mV} \cdot \mathrm{s}^{-1}$. a.) Cycles 1, 25, 50, 75 and 100. b.) First versus last cycle. c.) Two last cycles

Figure 5. Convergence assessment results for the Pt electrode. a.) Cycle-to-cycle convergence indicator. b.) Long term convergence indicator

Figure 6. Experimental cyclic voltammograms measured for the ceramic electrode (Type II CVs), at a scan rate of $\mathbf{1 0} \mathbf{m V} \cdot \boldsymbol{s}^{\mathbf{- 1}}$. a.) Cycles 1, 25, 50, 75 and 100. b.) First versus last cycle. c.) Two last cycles

Figure 7. Convergence assessment results for the ceramic electrode. a.) Cycle-to-cycle convergence indicator. b.) Long term convergence indicator 


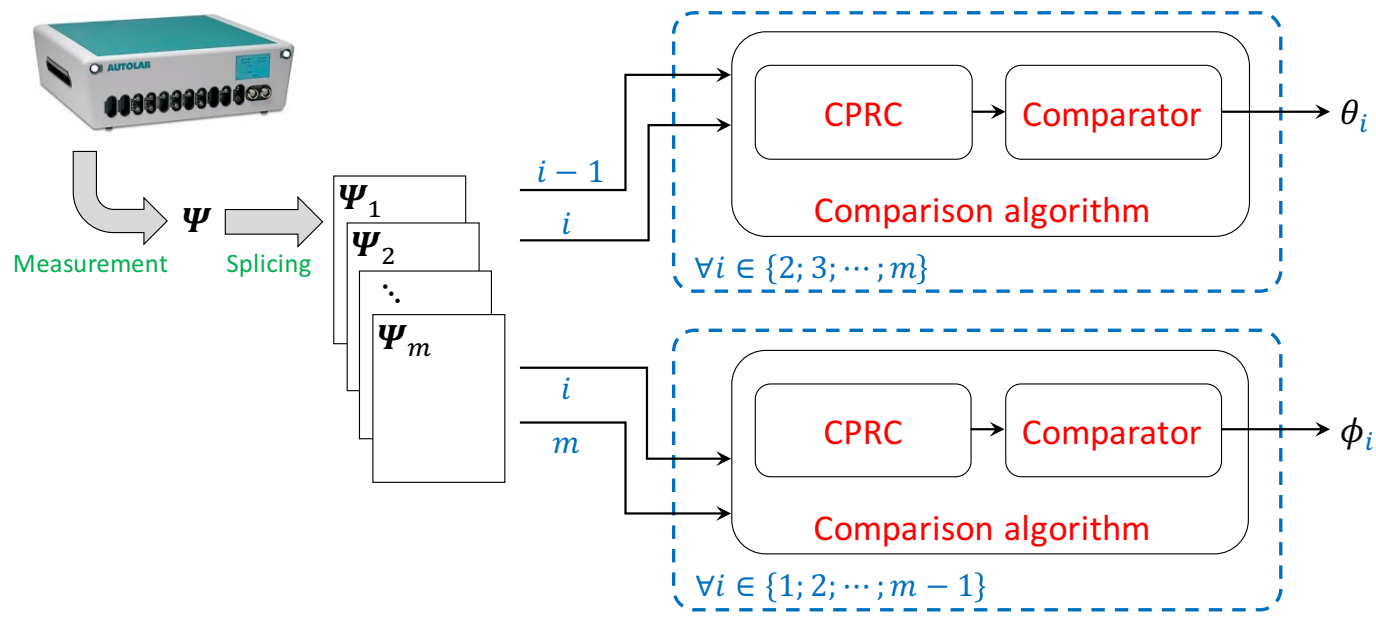

a.) Convergence assessment algorithm

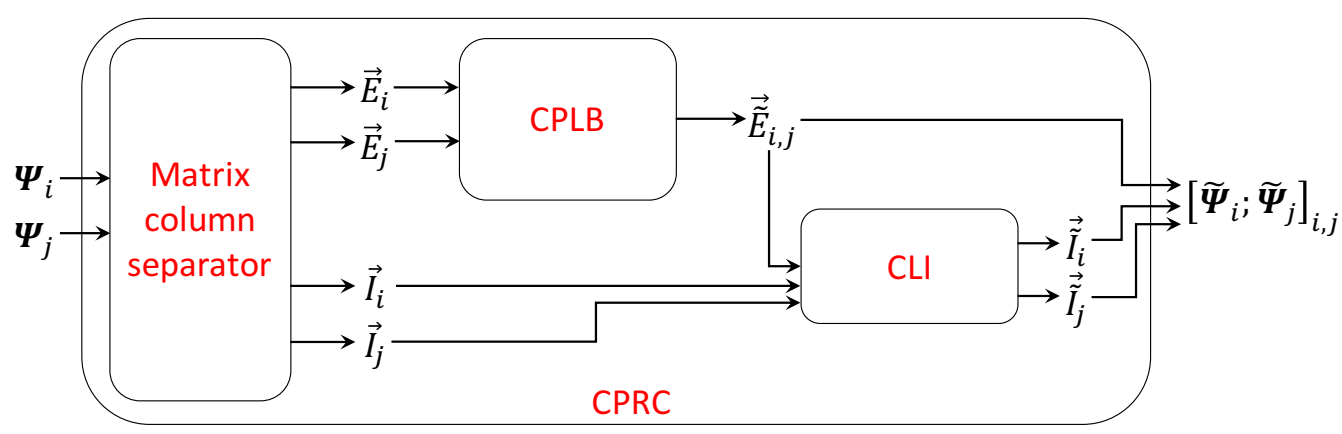

b.) Common potential range corrector routine

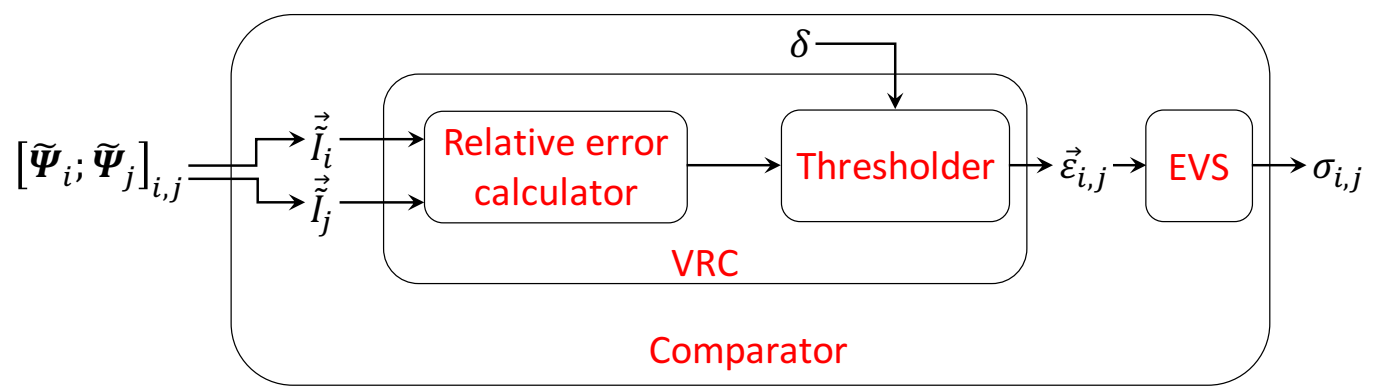

c.) Comparator routine

Figure 1. Convergence assessment algorithm block diagram 


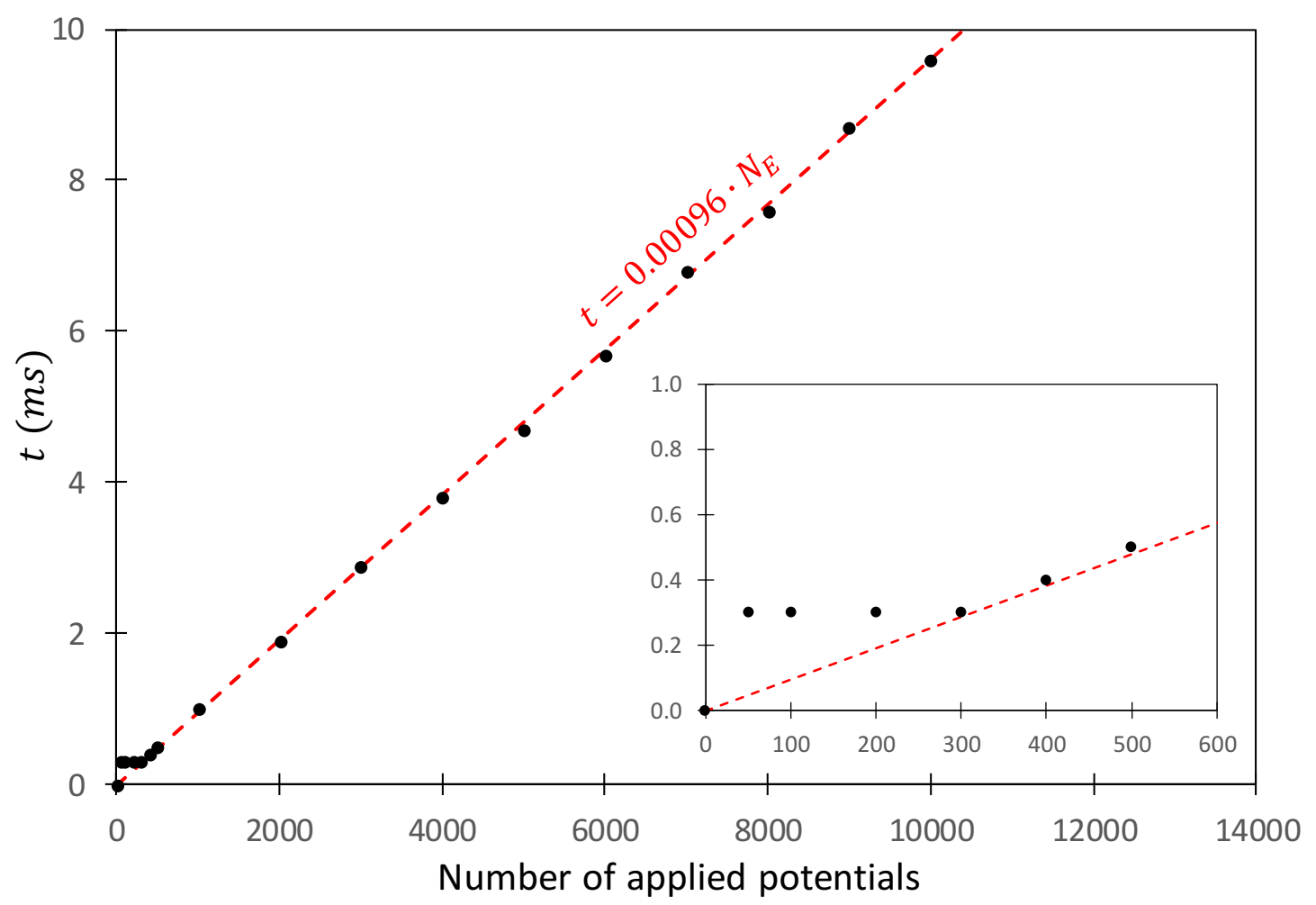

a.) Execution time as a function of the number of applied potentials

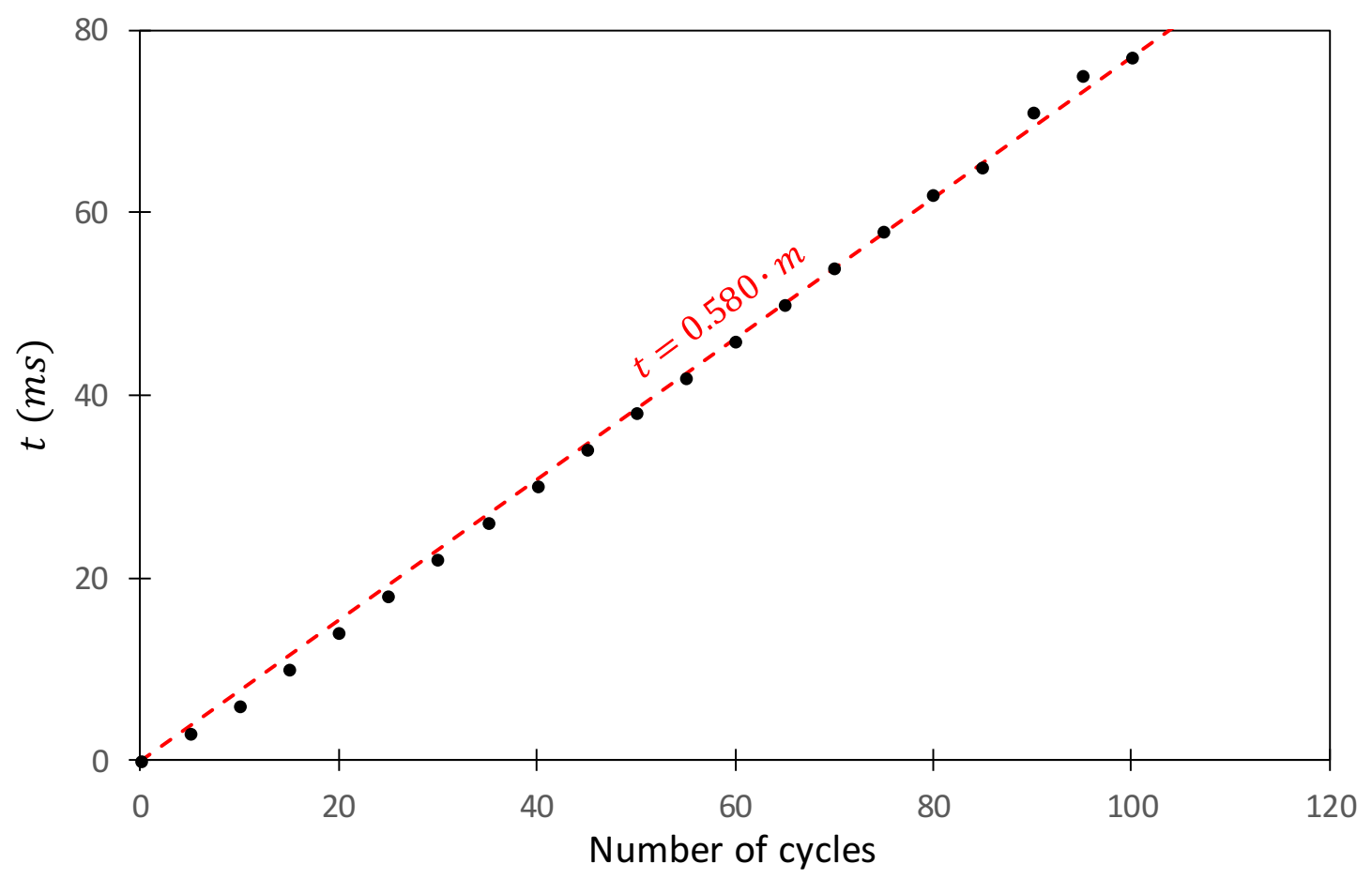

b.) Execution time as a function of the number of cycles of 600 applied potentials

Figure 2. Execution time of the convergence assessment algorithm 


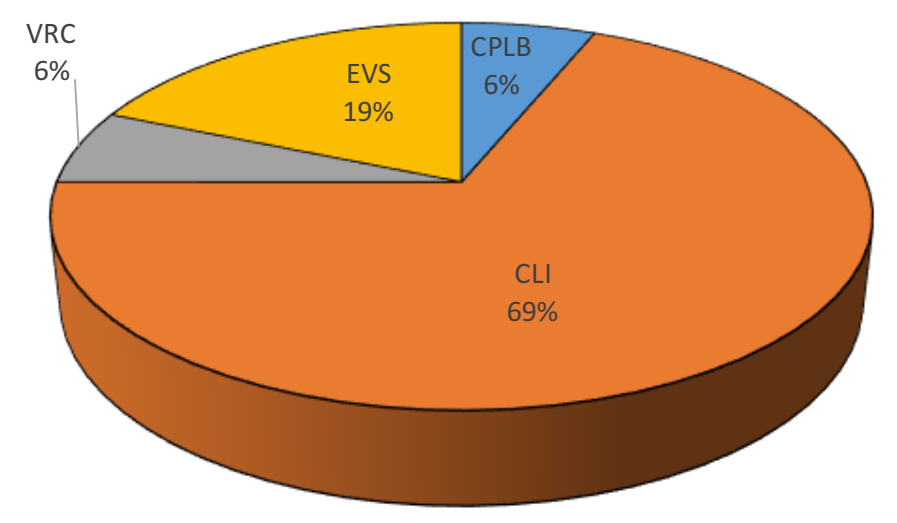

Figure 3. Computation time breakdown 


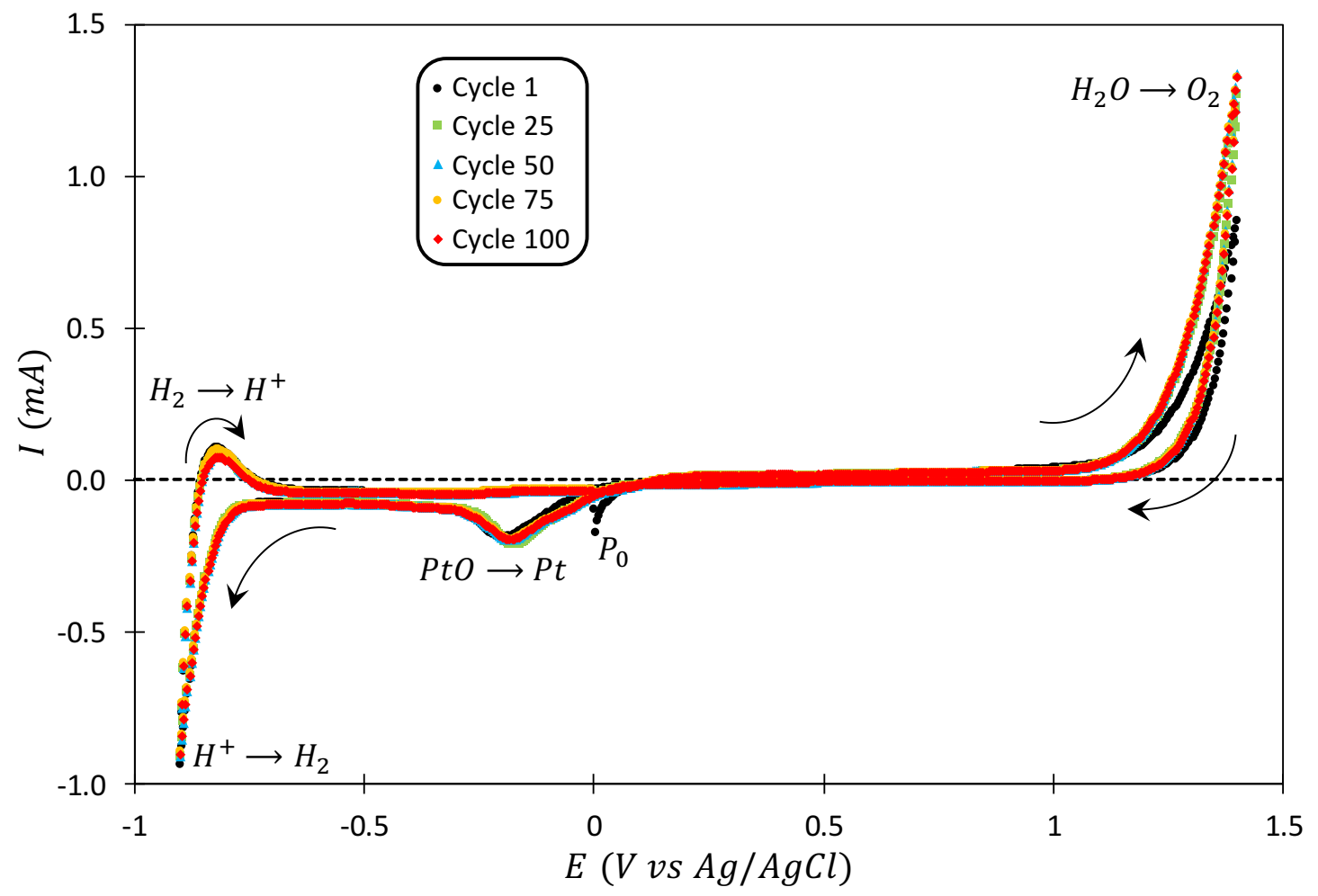

a.) Cycles 1, 25, 50, 75 and 100

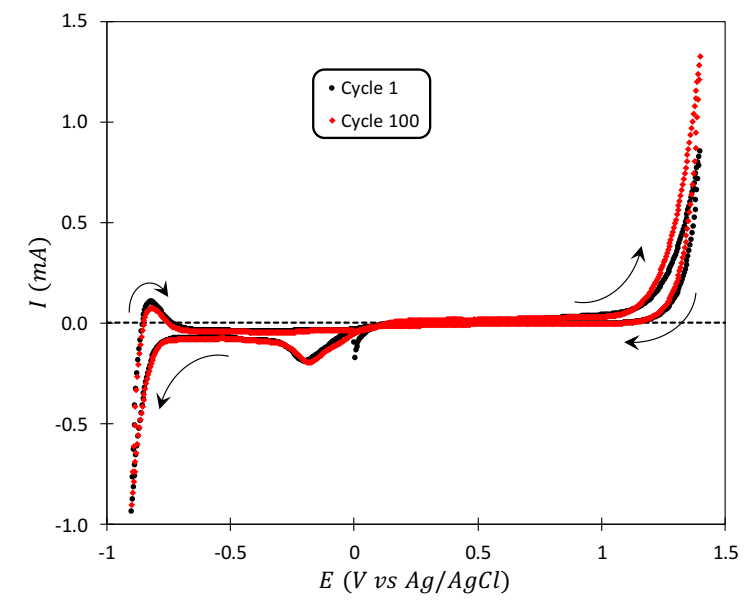

b.) First versus last cycle

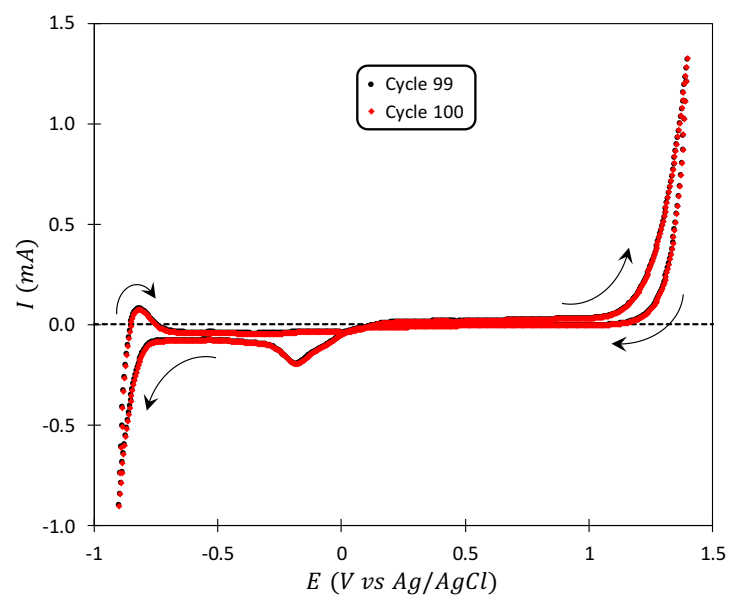

c.) Two last cycles

Figure 4. Experimental cyclic voltammograms measured for the Pt electrode (Type I $\mathrm{CVs}$ ), at a scan rate of $\mathbf{1 0} \mathrm{mV} \cdot \mathrm{s}^{-1}$ 


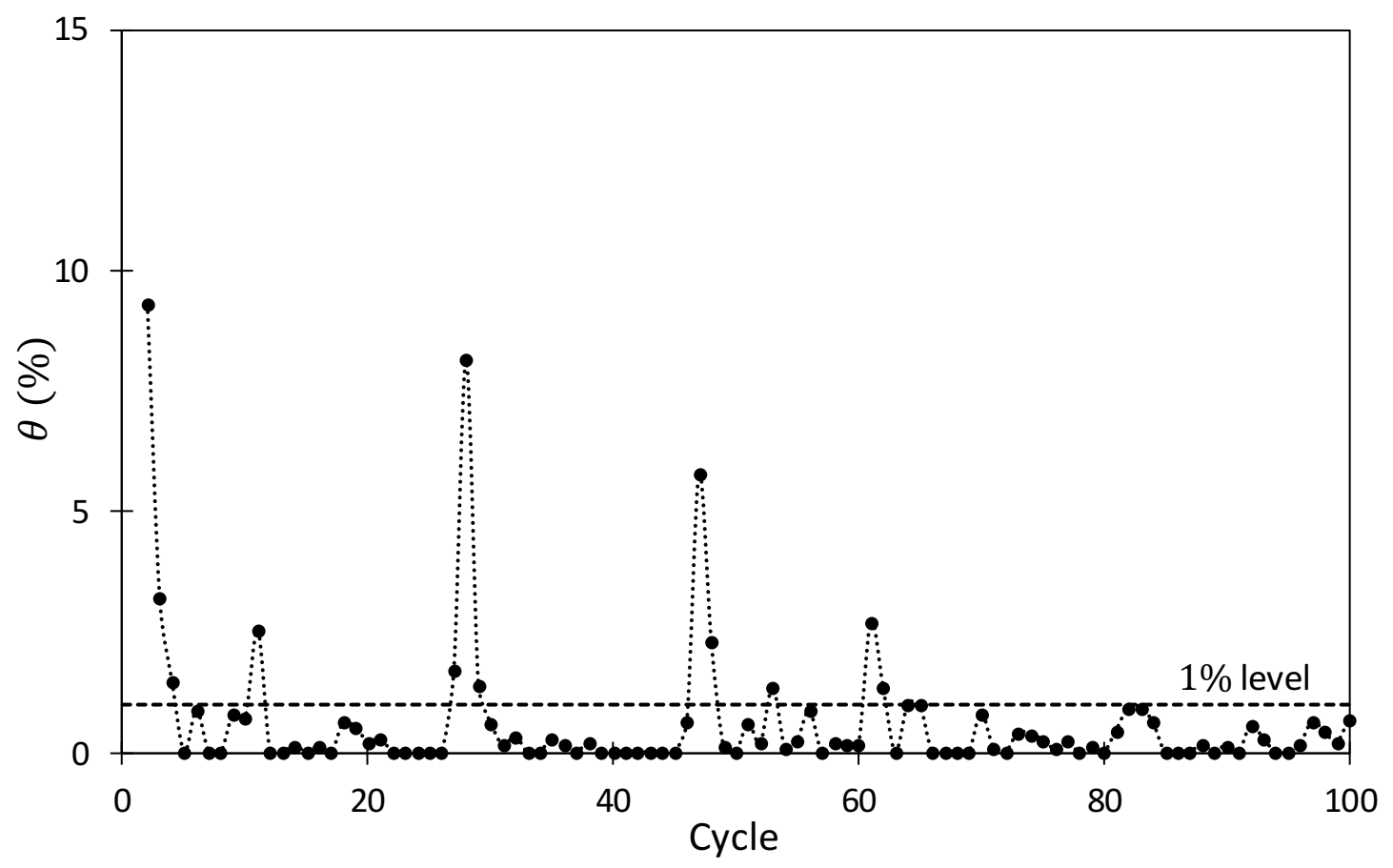

a.) Cycle-to-cycle convergence indicator

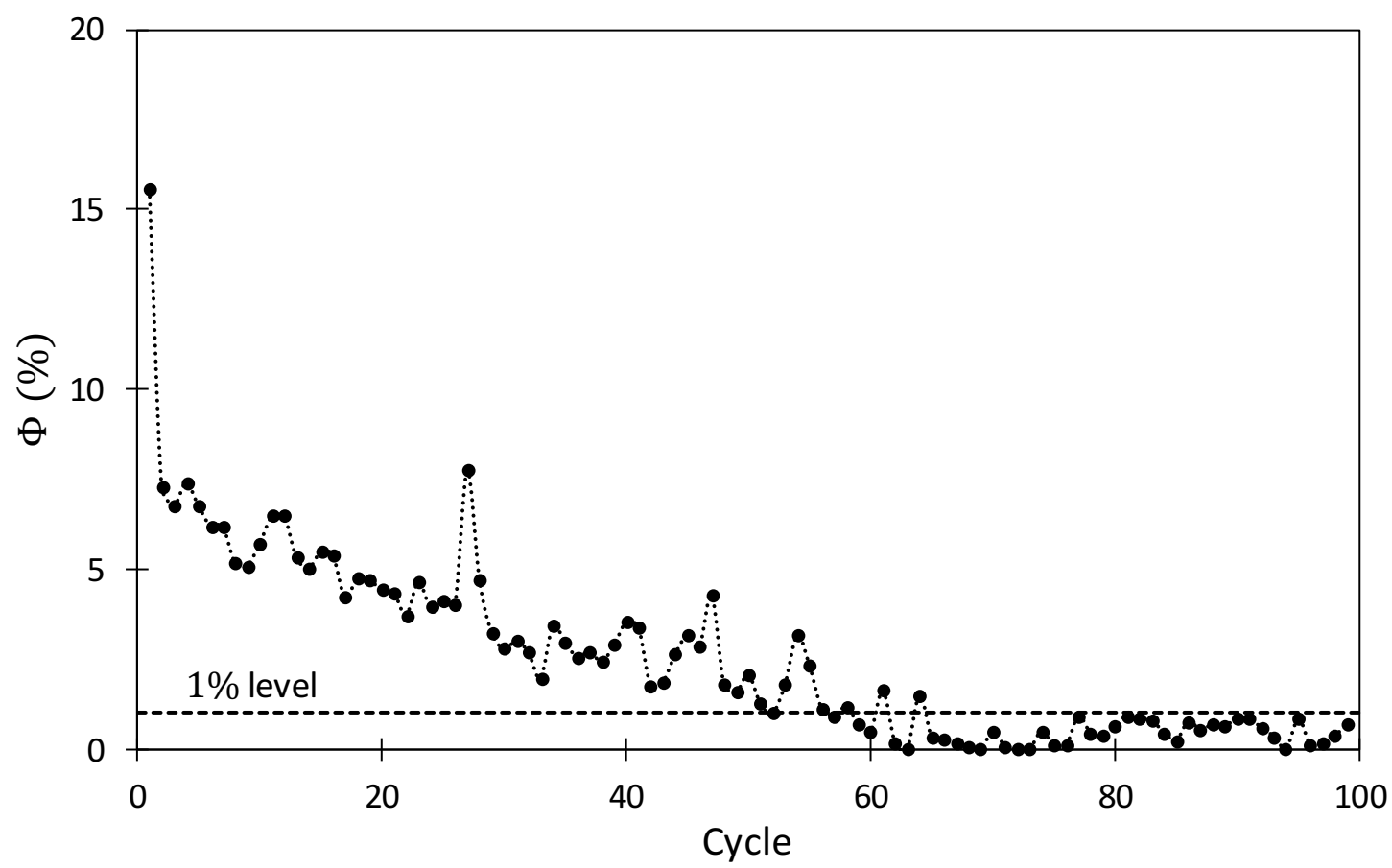

b.) Long term convergence indicator

Figure 5. Convergence assessment results for the Pt electrode 


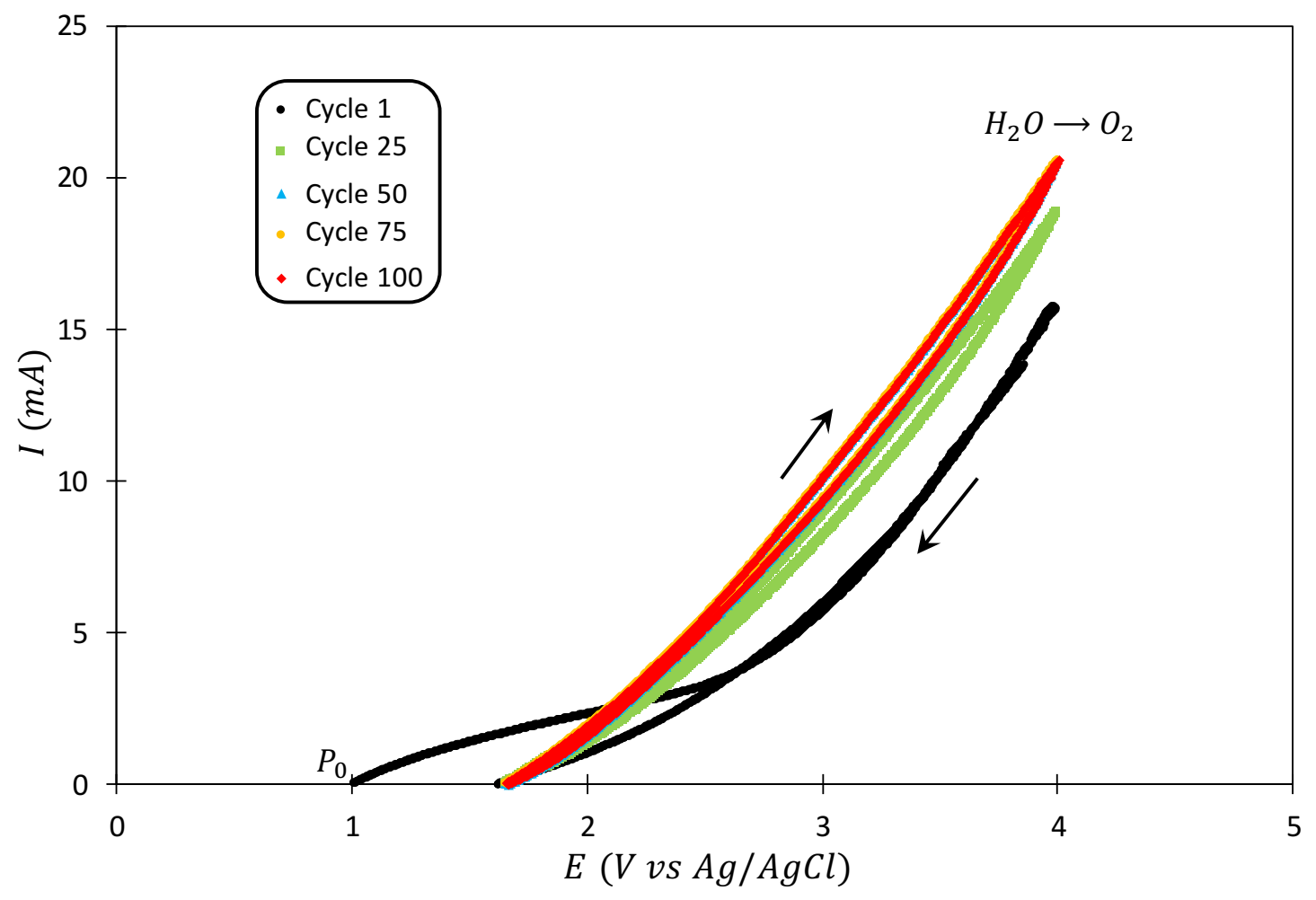

a.) Cycles 1, 25, 50, 75 and 100

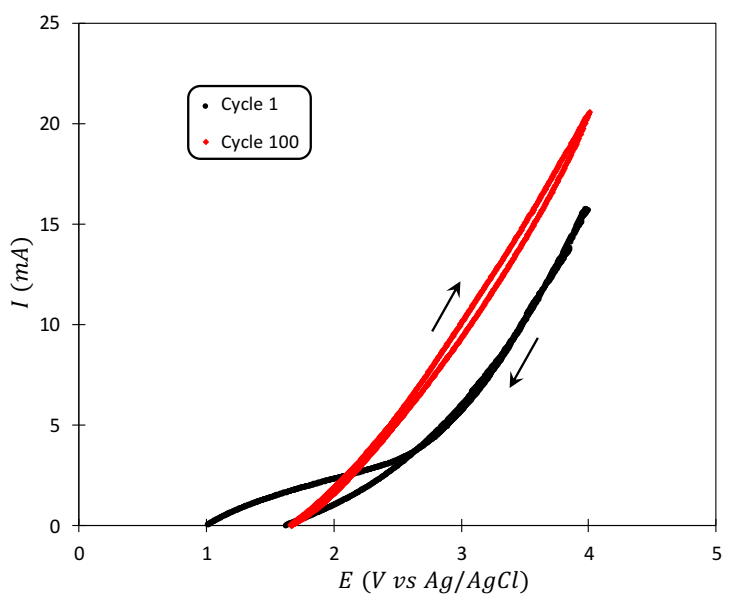

b.) First versus last cycle

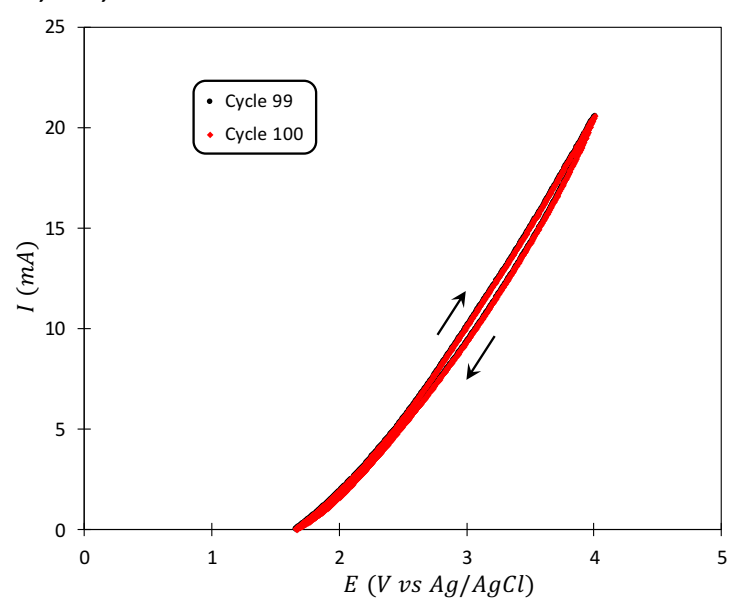

c.) Two last cycles

Figure 6. Experimental cyclic voltammograms measured for the ceramic electrode (Type II CVs), at a scan rate of $\mathbf{1 0} \mathbf{m V} \cdot \boldsymbol{s}^{-\mathbf{1}}$ 


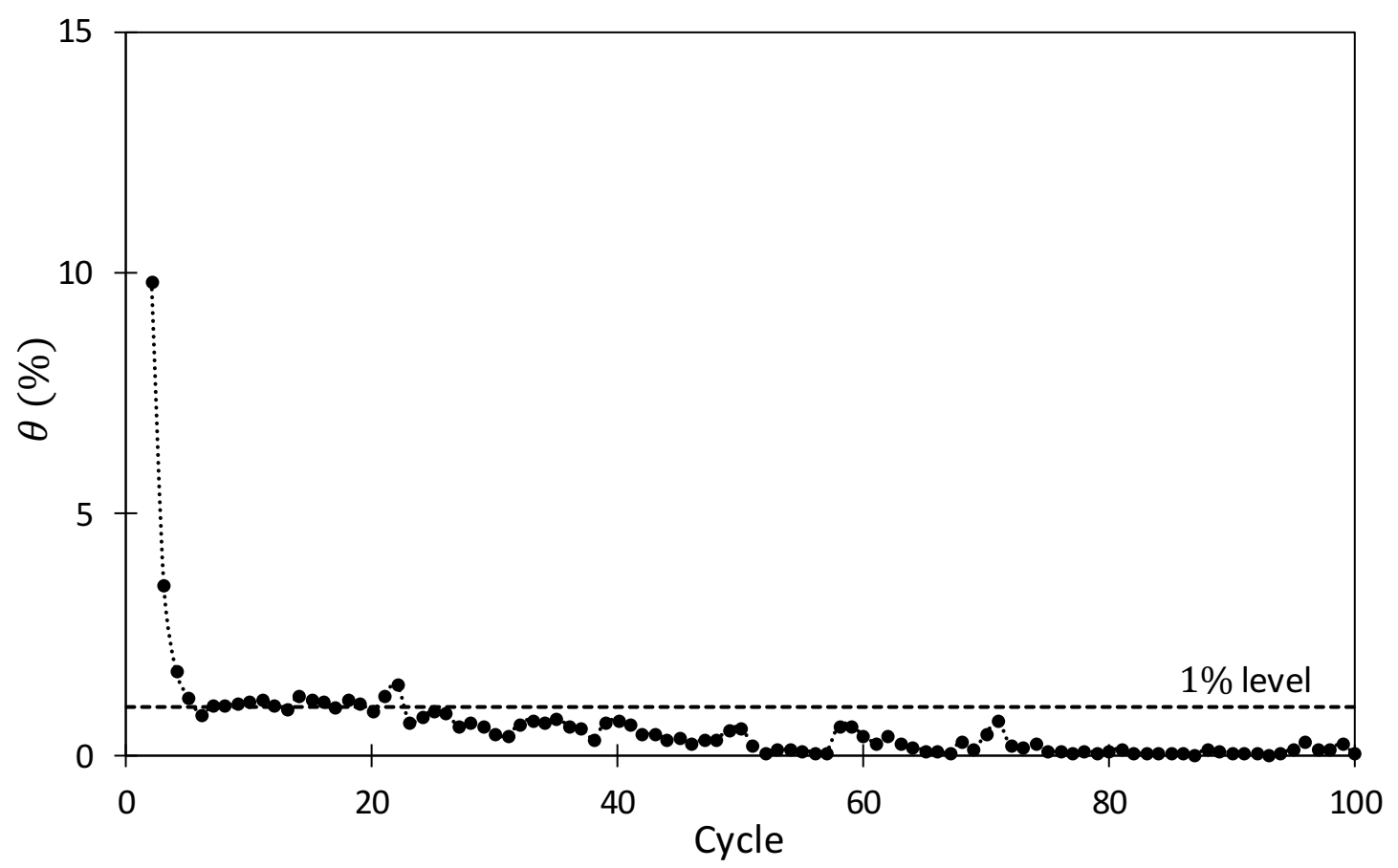

a.) Cycle-to-cycle convergence indicator

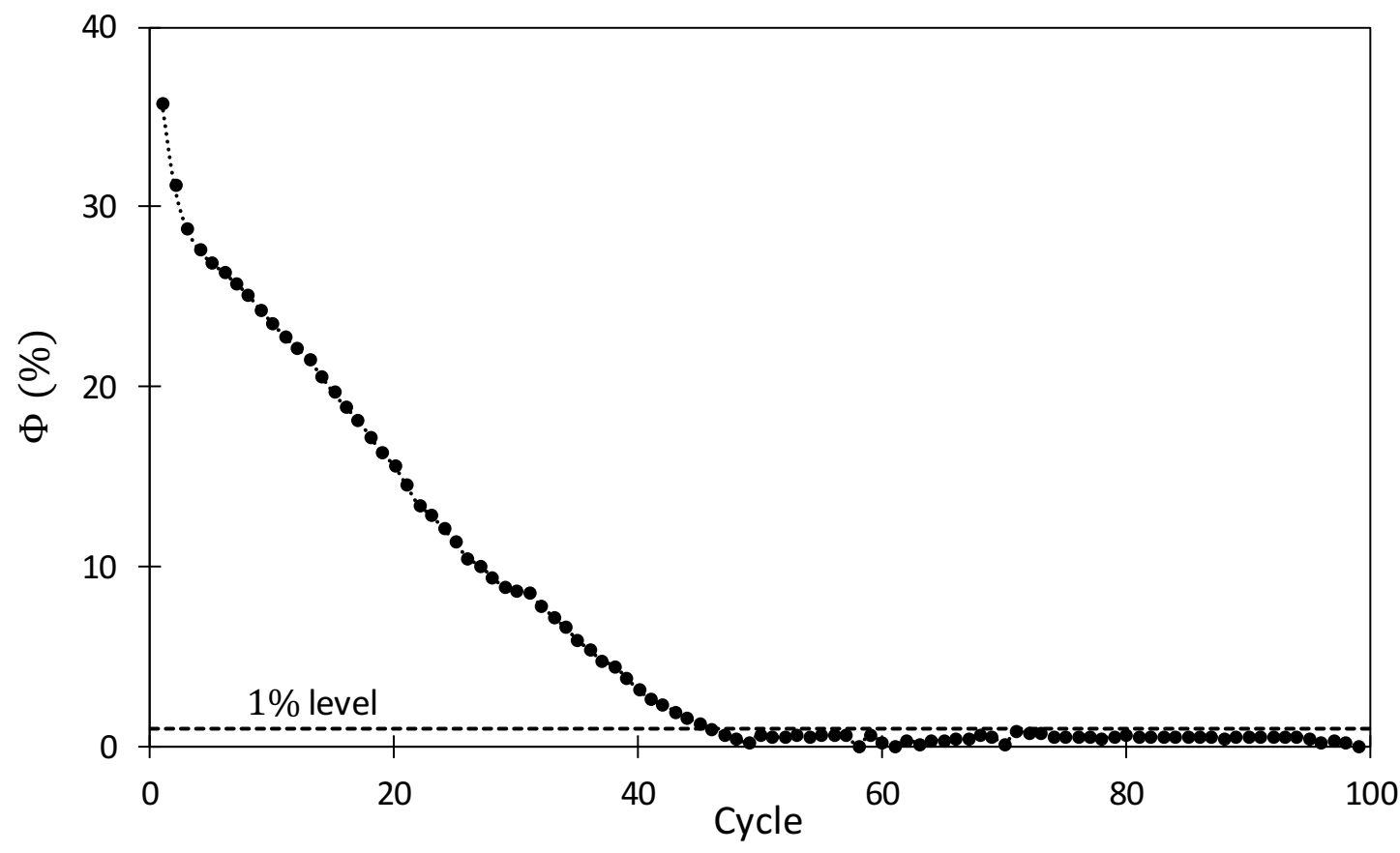

b.) Long term convergence indicator

Figure 7. Convergence assessment results for the ceramic electrode 